\title{
Software quality evaluation from research to industry: The Qseal Consortium approach
}

V. Asnaghi4, P. Caliman 3 , M. Campanail, W. Chiarottino 2, G. Di Capua6, F. Fabbrini 7, M. Fusani 7 , A. Jeanrenaud6, S. Mitolo1, D. Pina 5 , G. Rumi ${ }^{4}$, P. Salvaneschi ${ }^{5}$, I. Tsiouras $^{3}$

1-Cesvit, v.le Strozzi 1, 50129 Firenze (Italy), Tel. +39 (0) 55 4796425; Fax. +39 (0) 55 4796363; email: Campanai@fi.nettuno. it

2-Cimeco, v. Battistotti Sassi 11, 20138 Milano (Italy), Tel. +39 (0) 2 747601; Fax. +39 (0) 2719055

3-Etnoteam, v. A. Bono Cairoli 34, 20127 Milano (Italy), Tel. +39 (0) 2 261621, Fax: +39 (0) 26110755,

email:itsiuoras@etnoteam.it/pcaliman@etnoteam.it

4-IMQ, v. Quintiliano 43, 20138 Milano (Italy), Tel. +39 (0) 2 5073320;

Fax.+39 (0) 2 5073271; email: imqittl@icil64.cilea.it

5-Ismes, v.le Giulio Cesare 29, 20124 Bergamo (Italy), Tel. +39 (0) 35 307773; Fax. +39 (0) 35 211191;

email:psalvaneschi@ismes.it./dpina@ismes.it

6-Tecnopolis Csata Novus Ortus, strada provinciale per Casamassima Km. 3,70010 Valenzano, Bari (Italy), Tel. +39 (0) 80 8770111; email:dicapua@mailserv.csata.it/jeanrena@max.csata.it 7-IEI-CNR, v. S. Maria 46, 56126 Pisa (Italy), Tel. +39 (0) 50554095 email:fusani@iei.pi.cnr.it

\section{Abstract}

This paper presents the approach adopted by the Qseal Consortium with respect to software product quality evaluation and certification practices. This paper aims at giving an overview of the framework for evaluation and certification and describes the whole process, the main goals, and the available techniques.

\section{Keywords}

Software product assessment, product quality, evaluation and certification, product metrics and measures. 


\section{INTRODUCTION}

Over the past years both software engineers and users have paid increasing attention to assessing the quality of software products (Bache and Bazzana, 1993). This fact has lead many organisations to the development of practices enabling them to assess and give evidence of software quality degree that is suitable for its specified service (De Neumann and Bazzana, 1992).

Among others, many Italian companies have been involved in national and international projects (Robert, 1991), (TASQUE) concerning software quality evaluation and certification. Most of those companies increased their knowledge in real case studies and, starting from research experiences, developed proprietary measurement methodologies and apparatuses.

Due to the state of the art of software quality evaluation, each available measurement methodology and apparatus is a bit different from each other, and none of them can be considered the most suitable and credible.

In order to offer both to the developer, the customer and the user a harmonised service (measurement and apparatus) at the end of 1994 the Qseal Consortium was founded. The Consortium was constituted thanks to the effort of CIMECO (Centro Italiano MEtodologie di COntrollo), the Italian organisation for accreditation of certification bodies and testing laboratories in the IT\&T sector; it is composed by Etnoteam, Cesvit, Ismes, Tecnopolis Csata acting as testing laboratories (CNR participated as well as an external partner) and by IMQ acting as a certification body.

One of the main goals of the Consortium is to keep the activities transparent both to the providers and the users and in line with all the possible evolution of the state of the art; to this end, the Consortium established a few basic principles as its basis:

- participation is open to any company or individual being interested in its initiatives;

- it operates internationally and forster participation from all countries;

- it seeks harmonisation with similar schemes world-wide and will therefore liaise with standard bodies, ECITC and other harmonisation promoting committees.

The paper deals with the methodology and the measurement apparatus defined by the Qseal Consortium, and in particular defines:

- the software quality model;

- the measurement records, including information about how and where to measure;

- the interpretation functions.

\section{THE QSEAL APPROACH TO SOFTWARE QUALITY EVALUATION AND CERTIFICATION}

\subsection{The quality model}

What does the Qseal Consortium mean by quality? The first requirement is in fact a definition of quality that can be applied to software. It is the policy of the Consortium to seek harmonisation, and evaluate, and adopt (when appropriated) International Standards: so the Consortium has decided to adopt the definition provided in the ISO/IEC ISO 9126 (ISO/IEC International Standard 9126, 1991), which express the quality of a software product as a function of six quality characteristics: Functionality, Reliability, Efficiency, Usability, Portability and Maintainability. The characteristics are defined as follows:

- Functionality, that bears on the existence of a set of functions and their specified properties;

- Reliability, that bears on the capability of software to maintain its level of performance under stated conditions for a stated period of time; 
- Efficiency, that bears on the relationship between the level of performance of the software and the amount of resources used, under stated conditions;

- Usability, that bears on the effort needed for use, and on individual assessment of such use, by a stated or implied set of users;

- Portability, that bears on the ability of software to be transferred from one environment to another;

- Maintainability, that bears on the effort needed to make specified modifications.

However, this top level definition of quality is only a start; the ISO 9126 characteristics have been subdivided and refined into more specific sub-characteristics at lower levels which are relevant to the evaluation and certification activities.

The sub-characteristics presented by the ISO 9126 are not part of the standard; they are reported in Annex A and are "published to encourage use in practice and to collect experience for further edition".

Then, the evaluation of a software product will be done by assessing the above characteristics, and comparing the target values of specified characteristics of software with the values actually measured.

The decomposition of characteristics, as proposed by the ISO 9126, is presented in Figure 1.

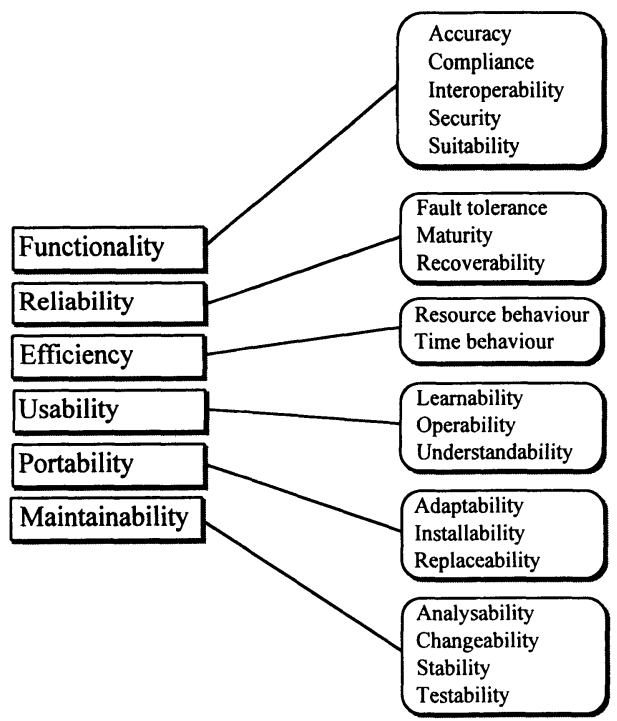

Figure 1 - Software Quality Model - ISO 9126.

\subsection{Quality views}

As far as evaluation and certification are concerned, one of the main problems refers to the fact that in many occasions, views of quality can be different according to the application domain; while the same general framework and the evaluation process can be applied, there are some product related aspects that can greatly differ: 
- quality profiles (that is the list of quality characteristics considered relevant for the software product);

- functionalities;

- part of the measurement apparatus.

To give an answer to this point the Consortium is about to organise a set of open Fora with the main goal to constitute the platform for promoting a common understanding on quality related issues among assessors, developers, and procurers of a specific industry (Di Capua and Jeanrenaud, 1994). The Fora are directed to gather different kind of organisations with a common interest and to define the quality profiles suitable for the particular kind of products, also dealing with specific functionalities and capabilities sought after in application of a specific market sector.

One of the key task for the Fora is to establish a set of predefined quality profiles to be associated to the Qseal quality mark; those who want to obtain the Qseal quality mark must agree on one of the predefined profiles, and the evaluation process must be in line with it.

\subsection{The evaluation process}

The evaluation process established by the Consortium is described as a step-wise procedure, which allows to express evaluation requirements in terms of quality characteristics as defined in ISO 9126. The evaluation covers various documents which can be considered as part of software product and uses a library of measurement records.

A measurement record is a structured encapsulation of software characteristics and evaluation techniques attached together. It identifies the product parts and elements of process information it needs and defines the elementary evaluation procedure and the format for reporting the measurements resulting from the application of the techniques.

After giving the answers to a set of checklist items, a suitable integration mechanism allows to assign values in a bottom-up fashion to sub-characteristics and characteristic. The integration mechanism is the following:

1. collection of basic measures;

2. evaluation of sub-characteristic values $s_{j}$

$s_{j}=\frac{\sum_{i} m_{i}}{n}$, computed on the applicable checklist items only $(n)$

3. evaluation of characteristic values $c_{k}$; they are computed starting from the $s_{j}$ values and combining them in different ways, using one of the average, minimum or maximum operators.

From a technical point of view, the evaluation process has been structured into few activities which are performed by the evaluator.

These activities, subdivided into 5 steps, are:

Step 1: analysis of the client requirements for evaluation in order to identify the purpose of the service provided and the quality profile; to release a trade mark the quality profile must correspond to one of the predefined (during the activities carried out within the Fora);

Step 2: specification of the evaluation whose aim is to identify product parts to be measured (that is documents to be provided to the evaluator), and define those characteristics to be evaluated for each part; 
Step 3: design of the evaluation which shall choose tools, instruments, and techniques and plan the valuation process (costs and time schedule);

Step 4: performing the evaluation that is measuring and testing selected product parts using the applicable measurement records;

Step 5: reporting on the results generated by the measurements activities; the final report shall be delivered to the client of the evaluation and in case of certification it shall have the Qseal trade mark on it.

It is quite important that, in order to be applicable and recognised by both producers and users, evaluation and certification activities should be:

- repeatable: repeated evaluation of the same product to the same evaluation specification by the same testing laboratory should produce the same results;

- reproducible: repeated evaluation of the same product to the same evaluation specification by different testing laboratory should produce the same results.

\section{The measurement records}

Once the quality characteristics are identified and refined into some more specific subcharacteristics at lower levels, the evaluation activities are performed and ratings are applied to each sub-characteristics; then the various values are combined and an aggregate score obtained.

Of course, the more the evaluation is subjective, the less it is repeatable and reproducible; a mature evaluation process should be based on objective measurements, rather than individual taste. For these reasons, an agreed upon set of measurement instruments, with guidelines for usage, has been produced; the step of selecting measurement instruments represented one of the key point for the whole process.

They have been selected according to the following criteria:

- to represent the most recent evolution of the state of the art;

- to be strictly connected to the sub-characteristic/characteristic they are associated;

- to be easily understandable;

- to be objectively applicable.

Characteristics, sub-characteristics, and measurement instruments are collected into Measurement records, composed by the following fields (Spinelli, Pina, Salvaneschi, Crivelli and Meda, 1995):

- Id: contains the checklist item identifier;

- Lev: contains the evaluation level to which the checklist item apply;

- Characteristic: contains the quality characteristic to which the checklist item apply;

- Sub-characteristic: contains the sub-characteristic to which the checklist item apply;

- Checklist item: contains the description of the measure to carry out;

- Notes: contains possible clarification concerned to the checklist item. Clarification are inserted only when necessary and applicable;

- How: this is the most important field: it contains the description of how to evaluate the checklist item. Generally it coincides with the mathematical formula to be applied.

- Answer: contains the interpretative function, which maps the possible answers to a standard range of merit (the interval $[0,1]$ ).

- Documents: contains the documents to which the checklist item is applicable.

Due to the characteristics of the measurement apparatus, a software product will be analysed, attributing each document available to one specific category (between parentheses some common denominations of documents in the category). The categories, reported in the 
following, have been subdivided according to: Development, Maintenance, and Quality assurance documentation.

-• Development

- RE Requirements (System/Software Requirement Specification);

- AR Architecture (System/Software Architecture);

- DD Detailed Design (Software Detailed Design);

- SC Software code;

- MC Machine code (Object code, Executable code);

- QD Qualification Documents (Test, Software qualification, System qualification requirements);

- TD Testing documents (Software Integration Plan, System Integrated Test Report);

- QD Qualification Documentation (Software Qualification Test Report);

- AT Acceptance testing and report (System Qualification Test Report, Acceptance Test Report;

- SI Software installation (Software Installation Plan);

- UMSoftware user's manuals;

-. Maintenance

- PI Post-installation data (Problem and Modification Report, Migration Plan, Withdrawal Plan);

-. Quality Assurance

- QA Quality Assurance (Quality Assurance Plan).

In selecting these documents, we started from the ISO 12207.2 standard (ISO/IEIC DIS 12207-1).

Two examples of measurement record are presented in Figure 2. The first one refers to Maintainability and the second one to Reliability. 

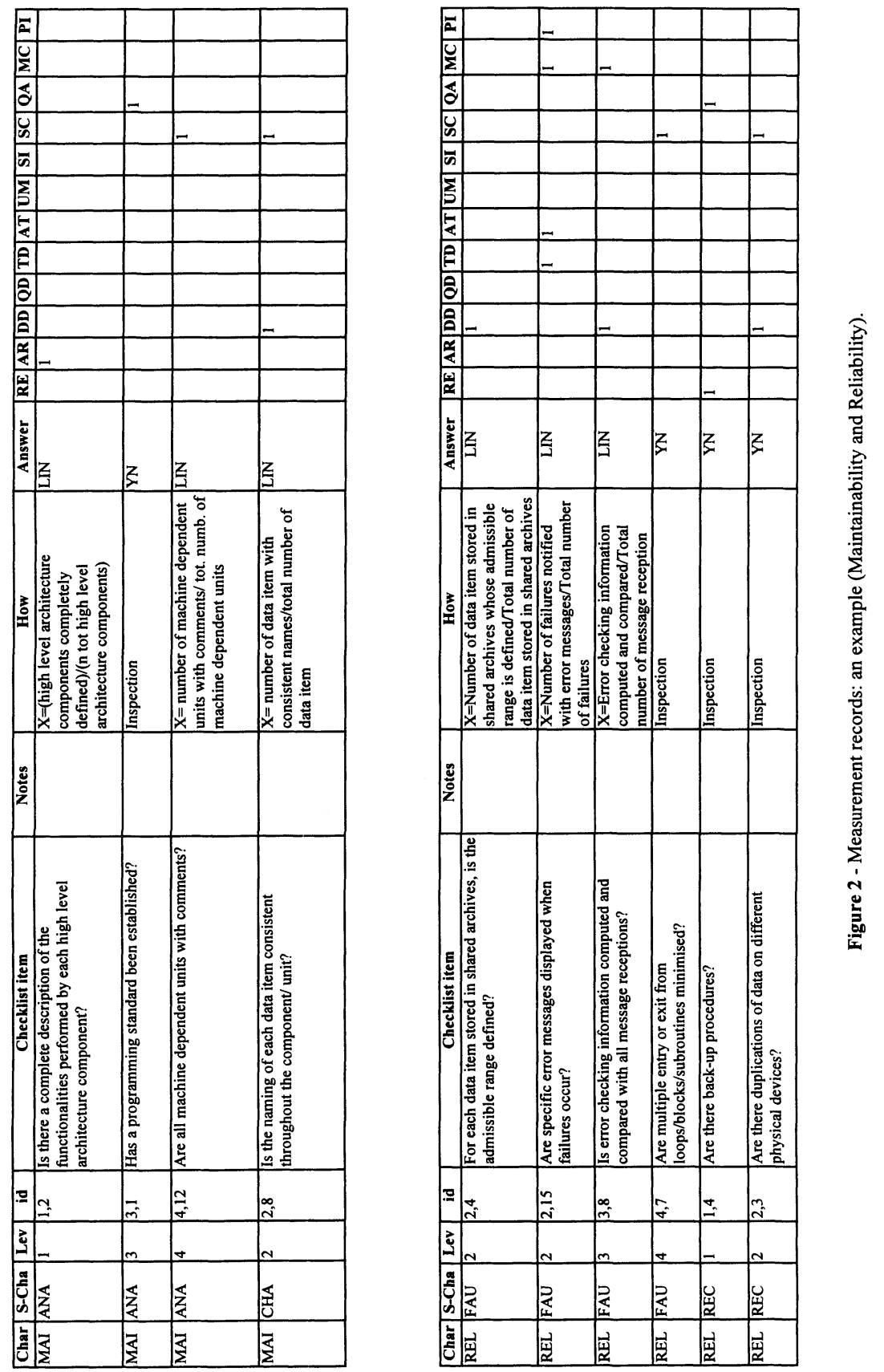


\subsection{The evaluation levels}

All the selected quality characteristics and sub-characteristics need not to be evaluated with the same degree of thoroughness for all types of applications. Nobody would expect the same effort to be allocated to the evaluation of a railway signal system, and a computer game.

To ensure this flexibility, the evaluation is level-oriented.

The Consortium has defined four levels (they constitute a hierarchy), which identify the depth of the evaluation: evaluation at different levels gives different degrees of confidence in the quality of the software product.

The evaluation levels can be chosen independently for each quality characteristic.

Now the question is: which criteria have been applied to select appropriate quality levels? Table 1 gives an overview of the criteria followed in defining the evaluation levels (ISO/IEC CD 9126-6, 1993); important aspects are those related to environment, to persons, to economy and to security.

\begin{tabular}{|cllll|}
\hline Level & \multicolumn{1}{c}{ Environmental } & \multicolumn{1}{c|}{ Personal } & Economic & \multicolumn{1}{c|}{ Security } \\
\hline 1 & Small damage to property & No risk to people & Negligible economic loss & No specific risk \\
\hline 2 & Local pollution & Few people injured & Significant economic loss & $\begin{array}{l}\text { Protection against } \\
\text { error risk }\end{array}$ \\
\hline 3 & $\begin{array}{l}\text { Recoverable } \\
\text { environmental damage }\end{array}$ & Threat to human lives & Large economic loss & $\begin{array}{l}\text { Protection of critical } \\
\text { data and services }\end{array}$ \\
\hline 4 & $\begin{array}{l}\text { Unrecoverable } \\
\text { environmental damage }\end{array}$ & Many people killed & Financial disaster & $\begin{array}{l}\text { Protection of } \\
\text { strategic data and } \\
\text { services }\end{array}$ \\
\hline
\end{tabular}

Table 1 - Guidelines for selecting the relevant evaluation levels

\subsection{The evaluation functions}

Evaluation function maps the possible answers to a standard range of merit: the interval $[0,1]$. Four evaluation functions have been selected; they are presented in the following together with their graphic explanation.
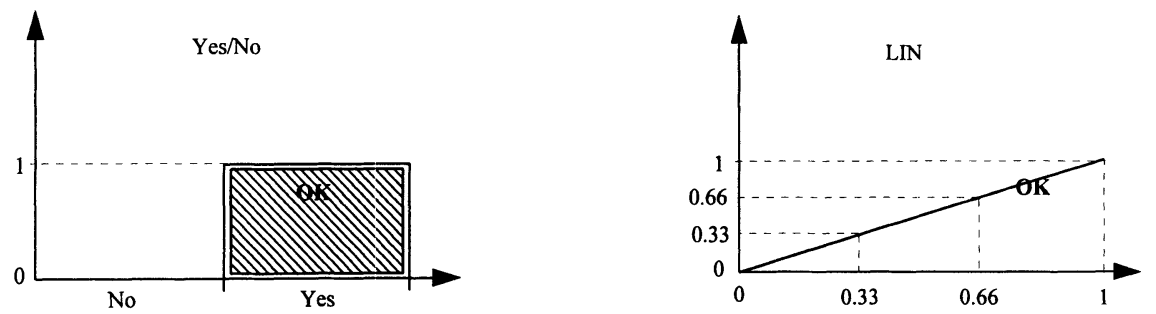

1. Yes/No is a function associated to checklist items whose possible answers is Yes or No. One example is: "Has a standard been established for the identification and placements of comments in the unit?". A positive result scores 1 , and a negative scores 0. 
2. The LIN function is associated to checklist items where there is a relation between two basic metrics, e.g. "Are I/O functions isolated from computational functions? (Calculate 1-(not isolated functions)/(tot. functions))". The best ratings are close to 1 .

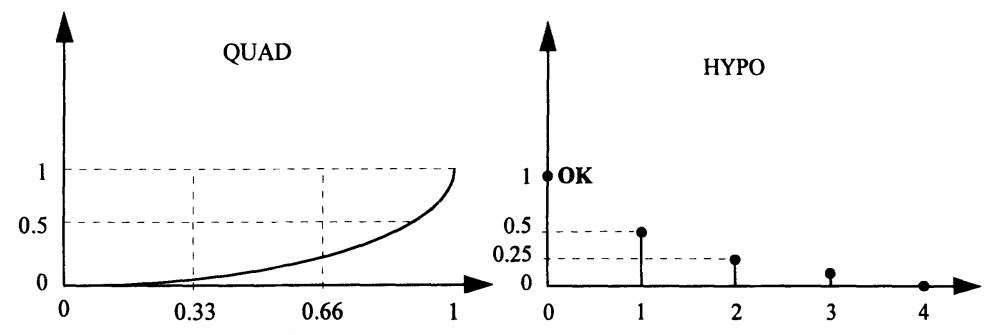

3. QUAD is a function similar to LIN. It differs from the previous, for it gives a worse rating to values even if they are close to the acceptable range; an example is the following "Are all components/ units independent of the source of the input and the destination of the output?"

Best ratings are close to 1 .

4. The fourth function, HYPO, is associated with the number of occurrence of a specified condition, e.g. "Is the number of loops in each component minimised?". The minimum, that is 0 scores 1; while the number of occurrences increase, the score diminish to $0.5,0.25$, and to 0 . Every number of occurrences superior to 4 scores 0 .

In the end, during the application of the measurement apparatus we faced a situations where constraints or technical aspects lead to have parameters which can have different values according to the specific application characteristics. We defined an other evaluation function, PAR, which depends on the value of one or two parameters.

One example of this function can be the following: "Is the ratio comments/statements well balanced?". As far as this checklist item is concerned, the values 0.33 and 0.66 can be suitable for an application in $\mathrm{C}$ language, but are meaningless for an application in Prolog.

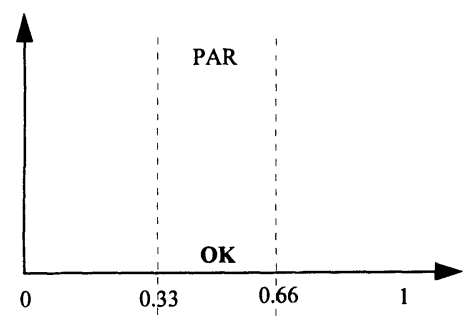




\section{Conclusion}

Why the Qseal Consortium approach should be a success?

We think that there are a few points that make it a success:

- the methodology and the tools take advantage of the last results in national and European research projects, and are quite close to the evolution of the normative.

- Each of the partners participated in national and European projects and the Consortium represent the effort to harmonise their experiences.

- The Consortium has, amongst its goals, to be as public as possible, in the sense that the Consortium aims at being an open organisation.

- The measurement apparatus has been designed to be as objective as possible to be easily applicable to evaluation and certification activities.

- The methodology, and the measurement apparatus have been designed to be easy to understand.

\section{REFERENCES}

Bache, R., Bazzana, G., Software Metrics for Product Assessment, McGraw Hill, London, December 1993

De Neumann, B., Bazzana, G., A methodology for the assessment/certification of software, 3rd European Conference on Software Quality, Madrid, November 1992

Robert, P., SCOPE, Achievements and Perspectives, Commission of the European Communities, Proceedings of Esprit Week, 1991

TASQUE - Eureka Project EU240, Tool for Assisting Software Quality Evaluation, TSQ/DEF/TECT7/10/1.2/0209/DEL

ISO/IEC International Standard 9126, Information technology - Software product evaluation Quality characteristics and guidelines for their use, International Standard Organisation, December 1991

Di Capua, G., Jeanrenaud, A., Italian perspective on IT assessment and certification agreement, Fourth European Conference on Software Quality, Basel, October 1994

Spinelli, A., Pina,D., Salvaneschi, P., Crivelli, E., Meda, R., Quality measurement of software products: an experience about a large automation system, Objective Quality 1995, Firenze

ISO/IEC DIS 12207-1, Information Technology - Software - Part 1: Software life-cycle process, International Standard Organisation,

ISO/IEC CD 9126-6, Guide to software product evaluation - The evaluator's guide, International Standard Organisation, 1993 